\title{
Multiple Algorithm Integration Based on Ant Colony Optimization for Endmember Extraction From Hyperspectral Imagery
}

\author{
Lianru Gao, Member, IEEE, Jianwei Gao, Jun Li, Member, IEEE, Antonio Plaza, Fellow, IEEE, Lina Zhuang, \\ $\mathrm{Xu}$ Sun, Member, IEEE, and Bing Zhang, Senior Member, IEEE
}

\begin{abstract}
Spectral unmixing is an important technique in hyperspectral image exploitation. It comprises the extraction of a set of pure spectral signatures (called endmembers in hyperspectral jargon) and their corresponding fractional abundances in each pixel of the scene. Over the last few years, many approaches have been proposed to automatically extract endmembers, which is a critical step of the spectral unmixing chain. Recently, ant colony optimization (ACO) techniques have reformulated the endmember extraction issue as a combinatorial optimization problem. Due to the huge computation load involved, how to provide suitable candidate endmembers for ACO is particularly important, but this aspect has never been discussed before in the literature. In this paper, we illustrate the capacity of ACO techniques for integrating the results obtained by different endmember extraction algorithms. Our experimental results, conducted using several state-of-the-art endmember extraction approaches using both simulated and a real hyperspectral scene (cuprite), indicate that the proposed ACO-based strategy can provide endmembers which are robust against noise and outliers.
\end{abstract}

Index Terms - Ant colony optimization (ACO), endmember extraction, hyperspectral imagery, multiple algorithm integration.

\section{INTRODUCTION}

$\mathbf{S}$ PECTRAL unmixing is one of the key techniques used in hyperspectral image processing and analysis. The basic models normally adopted in spectral unmixing are the

Manuscript received January 12, 2014; revised October 24, 2014; accepted November 04, 2014. Date of publication December 09, 2014; date of current version July 30, 2015. This work was supported in part by the Key Research Program of the Chinese Academy of Sciences (CAS) under Grant KZZDEW-TZ-18, in part by the National Natural Science Foundation of China under Grant 41325004 , and in part by the Interdisciplinary and Collaborative S\&T Innovation Research Team on Advance Earth Observation System, CAS. (Corresponding author: Lianru Gao.)

L. R. Gao, J. Gao, X. Sun, and B. Zhang are with the Key Laboratory of Digital Earth Science, Institute of Remote Sensing and Digital Earth, Chinese Academy of Sciences, Beijing 100094, China (e-mail: gaolr@radi.ac.cn; gaojw@radi.ac.cn; sunxu@ radi.ac.cn; zb@radi.ac.cn).

L. Zhuang is with the Key Laboratory of Digital Earth Science, Institute of Remote Sensing and Digital Earth, Chinese Academy of Sciences, Beijing 100094, China, and also with the University of Chinese Academy of Sciences, Beijing 100049, China (e-mail: 120015427@qq.com).

$\mathrm{J}$. Li is with the Guangdong Key Laboratory for Urbanization and GeoSimulation, School of Geography and Planning, Sun Yat-sen University, Guangzhou 510275, China (e-mail: lijun48@mail.sysu.edu.cn).

A. Plaza is with the Hyperspectral Computing Laboratory, Department of Technology of Computers and Communications, University of Extremadura, 10071 Cáceres, Spain (e-mail: aplaza@unex.es).

Color versions of one or more of the figures in this paper are available online at http://ieeexplore.ieee.org.

Digital Object Identifier 10.1109/JSTARS.2014.2371615 linear mixing model (LMM) and the nonlinear mixing model (NLMM) [1]-[3]. In LMM, it is assumed that multiple materials are spatially distributed as a checkerboard mixture in the instantaneous field of view (IFOV), and incident light interacts with only one material and the mixing occurs at the sensor. The mixing occurs in this case mainly because the hyperspectral sensor does not have enough spatial resolution to separate different pure spectral constituents (endmembers). On the contrary, nonlinear mixing occurs due to the fact that multiple materials in IFOV are distributed as an intimate mixture, and incident light encounter more than one material resulting in a mixed signature. In this case, the light is scattered by multiple materials before it is acquired by hyperspectral sensor. Due to mixture complexity, NLMMs generally are established for some specific objects. For example, Hapke and Shkuratov developed models which are consistently used for minerals [4], [5]. PROSPECT and SAIL have been developed for vegetation analysis [6], [7]. These models require prior knowledge of scene parameters, which are difficult to accurately obtain from a general processing perspective. Moreover, the nonlinear mixing process cannot be easily performed in mathematical fashion and requires additional information about the objects under analysis. Conversely, the LMM is simple to use in many real case studies. It has also been demonstrated that LMM is an acceptable and useful model in numerous different applications [1]-[3].

Over the past 20 years, several linear spectral unmixing algorithms have been developed [2]. In LMM, it is assumed that multiple scattering between endmembers is negligible. Therefore, the spectrum of each pixel can be approximated as a linear mixture of the endmember spectra, weighted by the fractional area coverage of each endmember within the pixel. Let $p$ be the number of endmembers in a hyperspectral imagery, and let $B$ be the number of channels. For a given pixel, the LMM can be formulated as follows:

$$
\mathbf{x}=\mathbf{M a}+\mathbf{w}
$$

where $\mathbf{x}$ is the $B \times 1$ spectrum vector of the mixed pixel, $\mathbf{M}=\left[\mathbf{m}_{1}, \mathbf{m}_{2}, \ldots, \mathbf{m}_{p}\right]$ is the $B \times p$ endmember matrix containing the signatures of pure material present in the covered area, $\mathbf{a}=\left[a_{1}, a_{2}, \ldots, a_{p}\right]^{T}$ is the $p \times 1$ vector denoting the fractional abundance of endmembers, and $\mathbf{w}$ is the $B \times 1$ vector indicating additive noise or modeling errors. If a hyperspectral image contains $N$ pixels, (1) can be extended in data 
matrix form as $\mathbf{X}=\mathbf{M A}+\mathbf{W}$, where $\mathbf{X}=\left[\mathbf{x}_{1}, \mathbf{x}_{2}, \ldots, \mathbf{x}_{N}\right]$, $\mathbf{A}=\left[\mathbf{a}_{1}, \mathbf{a}_{2}, \ldots, \mathbf{a}_{N}\right]$, and $\mathbf{W}=\left[\mathbf{w}_{1}, \mathbf{w}_{2}, \ldots, \mathbf{w}_{N}\right]$.

As mentioned above, $a_{i}(i \in\{1, \ldots, p\})$ denotes the fractional area coverage of the $i$ th endmember in the pixel. Two constraints are generally imposed in fractional abundance estimation according to (1). These are the abundance nonnegativity constraint (ANC) and abundance sum-to-one constraint (ASC), which can be defined as

$$
\begin{gathered}
\text { ANC }: a_{i} \geq 0, \quad \text { where } i=1, \ldots, p \\
\text { ASC }: \sum_{i=1}^{p} a_{i}=1 .
\end{gathered}
$$

When the endmember matrix $\mathbf{M}$ is given, the abundance matrix A can be calculated by a least squares method using

$$
\hat{\mathbf{a}}_{i}=\min _{\mathbf{a}_{i}}\left\|\mathbf{x}_{i}-\mathbf{M} \mathbf{a}_{i}\right\|_{2}^{2}, \quad \text { where } i=1,2, \ldots, N \text {. }
$$

Different least squares methods can be used based on which constraints are adopted. If all constraints are considered in abundance estimation, the corresponding algorithm is termed fully constrained least squares (FCLS).

The traditional hyperspectral unmixing processing chain contains three steps: dimensionality reduction, endmember extraction, and abundance estimation [1]. In LMM, the key task is to find an appropriate suite of endmembers [8]. Endmember extraction algorithms can be basically classified into two main categories: geometrical and statistical. Statistical methods mainly use parameter estimation techniques to extract endmembers, such as independent component analysis (ICA) [9], and Bayesian approaches [10]. Geometrical methods are generally based on convex geometry concepts. Due to different hypotheses considered, the geometrical methods can also be categorized into two types: pure pixel-based and minimum volume-based [2]. The minimum volume-based algorithms aim to find the simplex of $\mathbf{M}$ with minimum volume that includes all the observations. Among these algorithms, we can mention the simplex identification via split augmented Lagrangian (SISAL) [11], minimum volume simplex analysis (MVSA) [12], minimum volume-constrained nonnegative matrix factorization (MVCNMF) [13], minimum volume enclosing simplex (MVES) [14], or convex cone analysis (CCA) [15], among others.

In turn, the pure pixel-based algorithms seek endmembers under the assumption that they exist as pure pixels in the scene. Among these algorithms, we can mention the pixel purity index (PPI) [16], N-FINDR [17], iterative error analysis (IEA) [18], vertex component analysis (VCA) [19], simplex growing algorithm (SGA) [20], or sequential maximum angle convex cone (SMACC) [21], among many others. Additionally, several algorithms have included spatial information in addition to spectral information for endmember extraction. Examples include the automatic morphological endmember extraction (AMEE) [22] and the spatial spectral endmember extraction (SSEE) [23]. The spatial-spectral preprocessing (SSPP) can select a subset of spatially homogeneous and spectrally pure pixels from the hyperspectral image, thus enhancing the subsequent endmember extraction process [24]. Besides, sparse regression approaches supported by spectral libraries have also been widely used for linear spectral unmixing [25]-[27].

Despite the availability of many endmember extraction algorithms, in some studies, it has been pointed out that a single algorithm may not be able to provide the best answer due to several factors such as endmember variability [28]. From this viewpoint, ensemble solutions combining endmembers provided by different algorithms could provide a new way to approach the endmember extraction problem. For example, a classic and widely used algorithm such as PPI has been shown to be sensitive to noise. Similarly, N-FINDR and VCA are also easily affected by noise and outliers. To solve the aforementioned problems, in this paper, we explore the ability of intelligent methods with high optimal performance in order to combine the results provided by different endmember extraction algorithms. Specifically, we explore the feasibility of using ant colony optimization (ACO) [29] to overcome the aforementioned disadvantages. Experimental results indicate that the combination of endmember results produced by the ACO method is comparable or better than those found by individual algorithms such as N-FINDR and VCA. However, since the $\mathrm{ACO}$ is a random search algorithm, its computational speed is relatively slow. Consequently, candidate endmembers should be provided before ACO processing. It should be noted, however, that the computational performance of ACO has been largely improved using graphics processing units (GPUs) [30]. On the contrary, the design of an appropriate strategy to extract a suitable combination of endmembers using ACO has never been discussed.

In this paper, we design a multiple algorithm integration framework based on ACO in order to automatically extract endmembers from hyperspectral imagery. It consists of four parts: 1) estimation of the number of endmembers, 2) dimensionality reduction, 3) extraction of candidate endmembers, and 4) final endmember extraction. Commonly used methods, such as hyperspectral signal identification by minimum error (HySime) [31] and maximum noise fraction (MNF) [32], are adopted in this processing chain for determining the number of endmembers and reducing data dimensionality, respectively. The extraction of candidate endmembers is performed in this study using three highly representative algorithms: PPI, N-FINDR, and VCA, and the final endmembers are selected by ACO from the candidate endmembers produced by the aforementioned algorithms. The main reason for constraining our analysis to three endmember extraction algorithms: PPI, N-FINDR, and VCA in this work is that these algorithms are among the most widely used for endmember extraction purposes. Most importantly, we have found that the combination of these algorithms using ACO makes the endmember extraction process robust against noise and outliers. This point will be illustrated in our experiments. Also, it should be noted that ACO is an effective parallel strategy which can be efficiently implemented using high performance computing (HPC) techniques, which are now widely used in spectral unmixing applications [33]-[35]. It also presents an opportunity for advances in multimodal unmixing [2]. Therefore, the computational complexity of the ACO-based strategy developed in this paper is quite 
reasonable as it can be efficiently implemented in several HPC architectures.

The remainder of the paper is organized as follows. Section II describes the newly proposed multiple algorithm integration strategy based on ACO. In Section III, a quantitative experimental comparison of the proposed method with other methods using synthetic hyperspectral data is presented. Section IV evaluates the proposed method using real hyperspectral data acquired by the airborne visible infra-red imaging spectrometer (AVIRIS) on the cuprite mining district. Finally, Section V concludes with some remarks and presents several directions for future research.

\section{Multiple Algorithm Integration Based on ACO}

\section{A. Endmember Extraction Based on ACO}

The ACO algorithm [36], [37], targeted toward global optimization problems, is a representative swarm intelligence algorithm that utilizes artificial ants to imitate the way natural ants select routes. Ants in nature release a kind of chemical substance named pheromone on the routes when searching for a food source from their nest. The shorter the route is, the more pheromones are released. Each ant randomly selects a route according to the pheromone concentration of routes. That is to say, the routes with more pheromones are more attractive. Therefore, the routes with high pheromone concentration will be selected more and more; meanwhile, more pheromones are released on these routes. Pheromones indirectly play a role in exchanging "information" among ants in the search for food. Furthermore, pheromones on routes dissipate as time goes on. As a result, pheromones will gradually concentrate on the shortest route, which means that ants have found out the shortest route in a positive feedback way.

In our ACO algorithm for endmember extraction algorithm, extracting endmembers from hyperspectral data is regarded as an optimization problem [29], [30]. A directed and weighted graph, denoted by $G$, is utilized to generate the solution space and the heuristic information. Each vertex in $G$ corresponds to a pixel in the hyperspectral image. Artificial ants can release pheromones on the edges between vertexes. The weight of an edge in $G$ between vertex $v_{i}$ and vertex $v_{j}$, indicated by $\eta_{i j}$, is referred to as "visibility" that represents some essential characteristics of the solution space.

The route constructed by an artificial ant (i.e., a feasible solution) contains $m$ different vertexes in $G$, where $m$ is the number of endmembers. Each edge in $G$ is initialized with the same concentration of pheromones. Then, the artificial ants begin to search for food with the pheromones release on the route. When an ant arrives at the vertex $v_{i}$ after $t-1$ times, the probability of moving from $v_{i}$ to vertex $v_{j}$ is defined as

$$
p_{i j}(t)=\frac{\tau_{i j}^{\alpha}(t) \eta_{i j}^{\beta}}{\sum_{j \in \text { allowed }_{t}} \tau_{i j}^{\alpha}(t) \eta_{i j}^{\beta}} \quad \forall j \in \text { allowed }_{t}
$$

where allowed ${ }_{t}$ is the set of pixels that can be reached by the ant from $v_{i}$ (i.e., all pixels that have not been reached by the ant) at time $t$, and $\tau_{i j}(t)$ indicates the concentration of pheromones

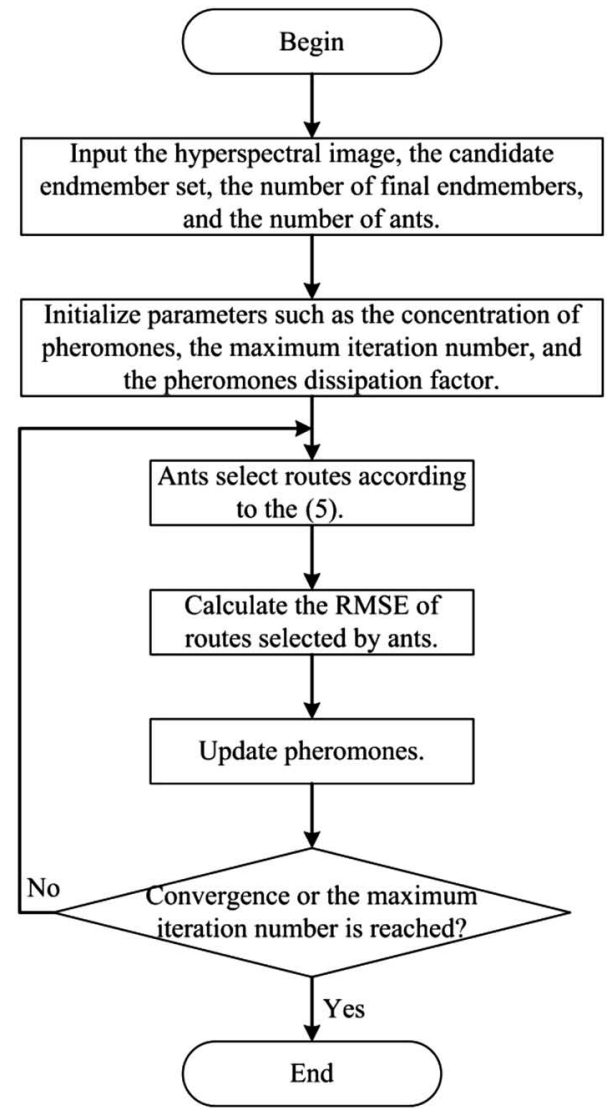

Fig. 1. Schematic diagram of ACO-based endmember extraction in the proposed strategy.

in the edge $\left\langle v_{i}, v_{j}\right\rangle$. Parameters $\alpha$ and $\beta$, respectively, denote the relative importance of pheromones and visibilities in the route selection. However, Ref. [30] demonstrated that unreasonable visibility can lead to a local optimal solution, and then it is better if $\beta$ was set to be 0 in this case.

The selected routes will be evaluated according to the objective function, i.e., the root-mean-square error (RMSE), after all ants complete their searching task in a given iteration. Pheromones on edges are updated as

$$
\tau_{i j}(t+1)=\rho \tau_{i j}(t)+\Delta \tau_{i j}(t)
$$

where $\rho$ is the pheromones dissipation factor and $\Delta \tau_{i j}(t)$ is the pheromones increment on the edge $\left\langle v_{i}, v_{j}\right\rangle$ at time $t$. If the minimum RMSE value in this iteration is $f_{t}$ and its corresponding path is route ${ }_{t}$, then $\Delta \tau_{i j}(t)$ is

$$
\Delta \tau_{i j}(t)=\left\{\begin{array}{cl}
Q / F\left(f_{t}\right) & <v_{i}, v_{j}>\in \text { route }_{t} \\
0 & <v_{i}, v_{j}>\notin \text { route }_{t}
\end{array}\right.
$$

where $Q$ is a constant that controls the value of pheromone adjustments $\Delta \tau_{i j}(t)$, and $F$ is a function of $f_{t}$ which can ensure that the better $f_{t}$ is, the more pheromones are released.

After the iteration at time $t$ has been completed, the ants go to the next iteration for searching the optimal solution. The algorithm stops when the same optimal route is obtained in several consecutive iterations, which is considered as a convergence state, or the iterations number reaches a predetermined 


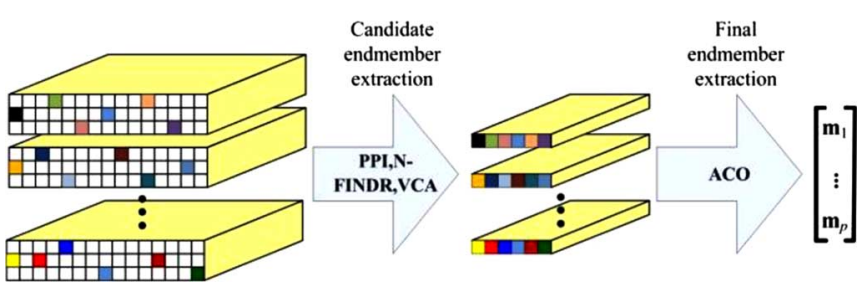

Fig. 2. Diagram of the approach for endmember extraction using multiple algorithms.

maximum number. For illustrative purposes, Fig. 1 shows a schematic overview of ACO-based endmember extraction in the proposed strategy.

\section{B. Multiple Algorithm Integration: Strategy Design}

The purpose of our multiple algorithm integration framework is to take adequate advantage of different algorithms, and effectively combine them together, which brings robustness against noise and outliers. A similar approach has been successfully used in target detection and image classification for hyperspectral imagery [39], [40]. In this paper, we design a multiple algorithm integration strategy for endmember extraction purposes. Obviously, it is very important to design a practical strategy and select appropriate algorithms for it.

The integration strategy developed in this paper is developed under the assumption that pure pixels exist in hyperspectral images. Although there are many algorithms developed without this assumption, our main goal in this work is to illustrate the advantages of ACO in the task of integrating the results provided by other endmember extraction algorithms. For this reason, we have decided to constrain ourselves to the pure pixel case and use three highly representative algorithms in this category: PPI, N-FINDR, and VCA. Several studies have suggested that these algorithms are sensitive to the noise or outliers [8], [29], [41]. In turn, ACO has been shown to be insensitive to noise in the task of endmember extraction [29]. However, ACO itself is computationally complex. Based on these observations, our introspection in this work is that traditional endmember extraction algorithms and ACO exhibit complementary features. Therefore, our goal is to design a multiple algorithm integration strategy combining the advantages of these algorithms. The strategy should be robust, insensitive to noise and outliers, and computationally efficient. The performance of the proposed framework (based on these algorithms) will be comparably analyzed by experiments with synthetic and real hyperspectral data.

It is important to emphasize that, in order for the proposed strategy to work, the number of candidate endmembers should be higher than the number of final endmembers extracted by ACO. For the PPI, we can control this by setting a threshold $t$ to get a specific number of endmembers. In order to address this issue with N-FINDR and VCA, we divide the input hyperspectral scene into a set of $K$ subblocks on a line-by-line basis, as illustrated in Fig. 2. The data partitioning strategy is the socalled spatial-domain partitioning as suggested in [42], where every pixel vector (spectral signature) is assigned entirely to the

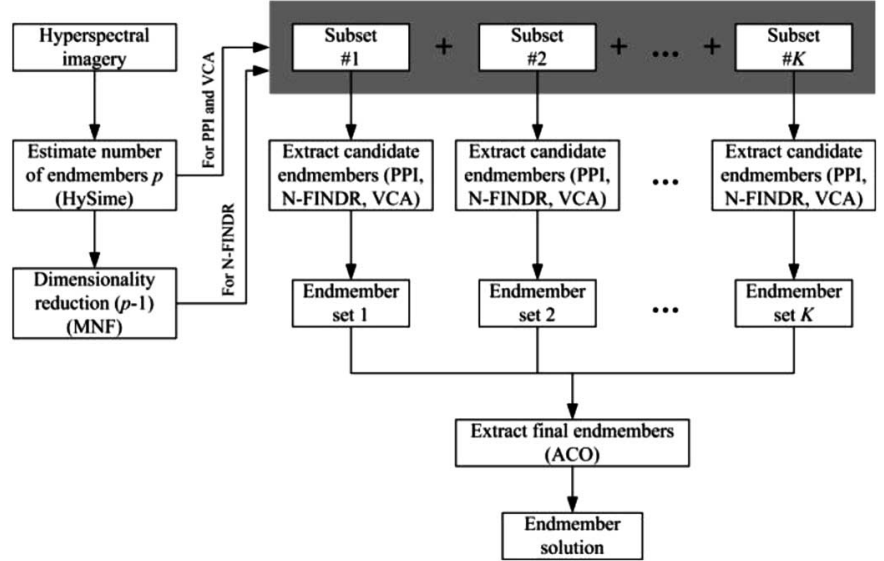

Fig. 3. Flowchart of our proposed multiple algorithm integration strategy based on ACO.

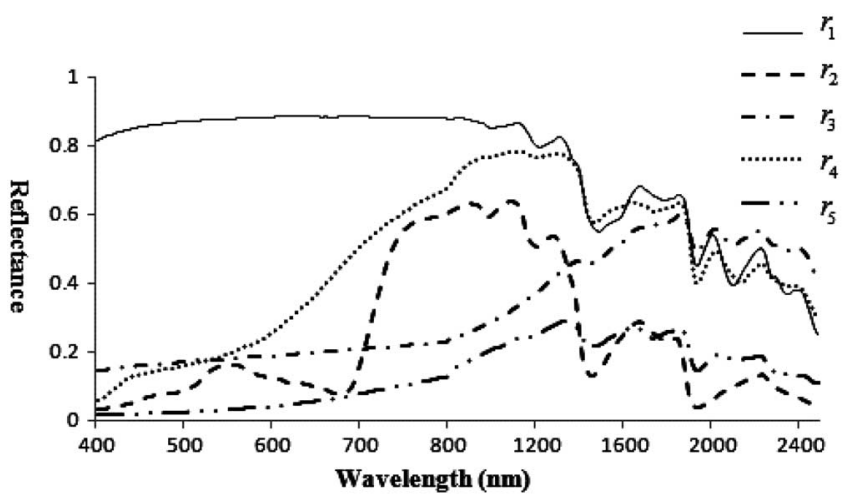

Fig. 4. Reflectance spectra used in the synthetic image simulation. All of these spectra are selected from veg_1dry.sli spectral library in ENVI software, where $r_{1}$ is Spruce Cellulose, $r_{2}$ is Mormon Tea, $r_{3}$ is Bigberry Manzanita, $r_{4}$ is Sycamore, and $r_{5}$ is White Peppermint.

same subblock for spatial and spectral data integration. Then, N-FINDR and VCA are applied to each subblock in order to extract endmembers, and the results from all subblocks are collected together to provide candidate endmembers for ACO. The number of candidate endmembers can be controlled by the number of subblocks. To make the multiple algorithm integration strategy uniform and standardized, image partitioning is thus considered as a prerequisite step for PPI, N-FINDR, and VCA. Similar partitioning strategies have been adopted in previous studies [23], [43]. In addition, this strategy can be easily implemented in parallel [44], [45]. Such subblock processing is also quite suitable when images cover large areas [2]. Note that this subblock processing is just an efficient way to find out suitable candidate endmembers for ACO instead of using all pixels as candidates. So, it contributes more to the efficiency and less to the accuracy, which would be discussed in more detail in Section III-C.

For illustrative purposes, a diagram depicting our proposed multiple algorithm integration strategy is given in Fig. 3. As shown by the diagram, HySime is adopted in our strategy to estimate the number of endmembers $p$ contained in the original hyperspectral image. It provides a requisite parameter for automatically processing the data. For instance, this avoids missing important endmembers by making sure that, $p$ endmembers 


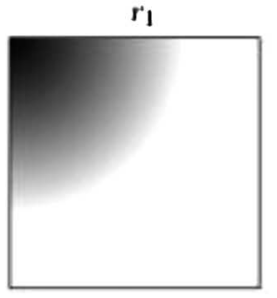

(a)

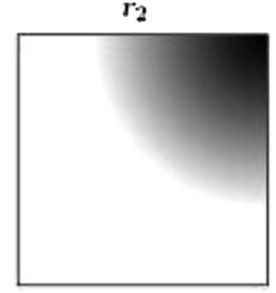

(b)

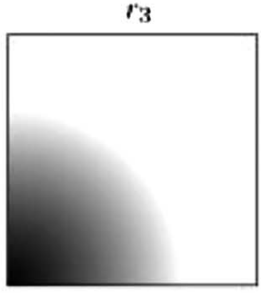

(c)

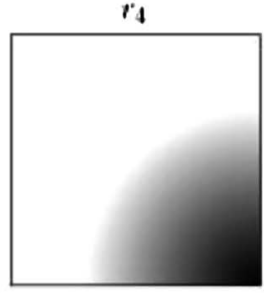

(d)

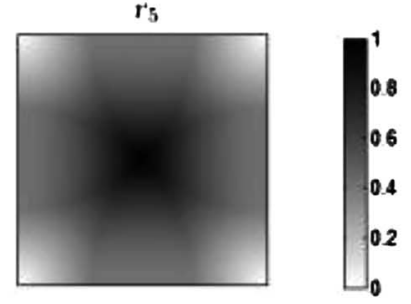

(e)

Fig. 5. Fractional abundance distributions used in image simulation for different selected spectra.

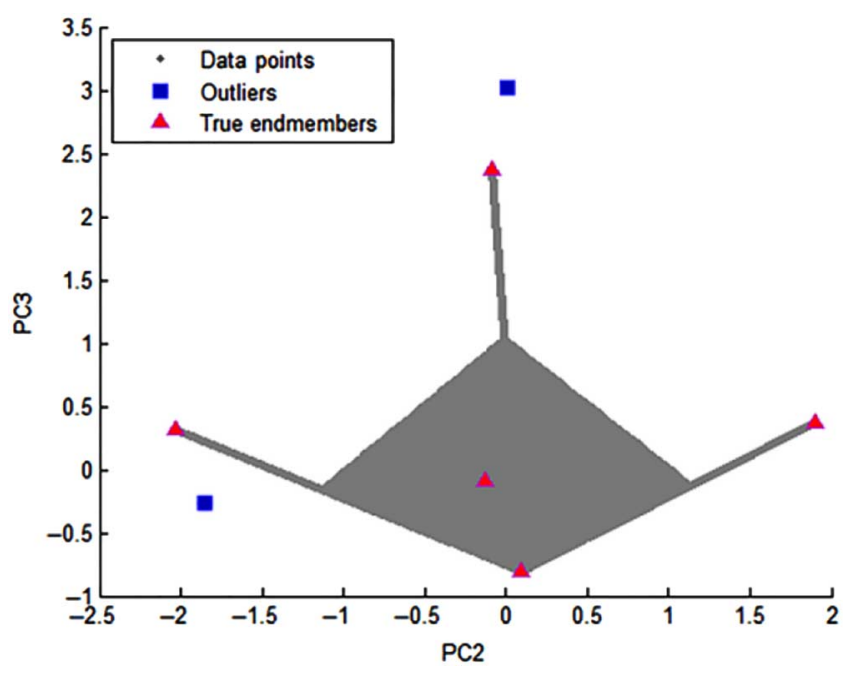

Fig. 6. Simulation of outliers in our synthetic data.

should be extracted from each subblock. To meet the dimensionality requirements of N-FINDR, MNF is also used as an important processing block in our strategy. In our procedure, HySime is always the first step. If PPI and VCA are chosen, the image partition will be the second step. Otherwise, MNF is implemented as the second step for N-FINDR before image partitioning, which is used in this context to subdivide the image cube into multiple blocks made up of entire pixel vectors. Then, candidate endmembers are extracted from each subblock using PPI, N-FINDR, and VCA. All of endmembers in each subset are collected together, and $(K \times p)$ candidate endmembers are obtained by means of this procedure. Finally, ACO is applied to extract the final set of endmembers.

\section{EXPeriments With Synthetic DatA}

\section{A. Synthetic Data Simulation}

A synthetic hyperspectral data set has been simulated using $p=5$ endmembers with specific abundance maps. These endmembers, namely $r_{1}, r_{2}, r_{3}, r_{4}$, and $r_{5}$, are selected from veg_1dry.sli spectral library in ENVI software. The spectra of these five endmembers are shown in Fig. 4. The simulated pixels have $B=206$ spectral bands ranging from 0.4 to $2.5 \mu \mathrm{m}$. The spatial size of synthetic data is $200 \times 200$ pixels. Four spectral signatures, $r_{1}, r_{2}, r_{3}$, and $r_{4}$ are arranged at the vertices of the simulated image. Signature of $r_{5}$ is located at the central pixel. As shown in Fig. 5, the abundance of each endmember
TABLE I

Average Spectral Angle Scores (in Degrees) Between the SPECTRAL IN Fig. 4 AND THEIR CORRESPONDING ENDMEMBERS EXtracted by SEVERAL Multiple Algorithm InTEgration STRATEgIES From the SynthetiC SCENES

\begin{tabular}{c|c|c|c|c|c|c}
\hline $\begin{array}{c}\text { Candidate } \\
\text { endmembers }\end{array}$ & $\begin{array}{c}\text { Endmember } \\
\text { extraction }\end{array}$ & $\begin{array}{c}\text { SNR= } \\
\mathbf{2 0 : 1}\end{array}$ & $\begin{array}{c}\text { SNR= } \\
\mathbf{4 0 : 1}\end{array}$ & $\begin{array}{c}\text { SNR= } \\
\mathbf{6 0 : 1}\end{array}$ & $\begin{array}{c}\text { SNR= } \\
\mathbf{8 0 : 1}\end{array}$ & $\begin{array}{c}\text { SNR= } \\
\mathbf{1 0 0 : 1}\end{array}$ \\
\hline \multirow{5}{*}{-} & N-FINDR & - & $\mathbf{1 . 6 8 9}$ & 0.179 & $\mathbf{0 . 0 1 8}$ & $\mathbf{0 . 0 0 2}$ \\
\cline { 2 - 7 } & PPI & - & - & 0.181 & $\mathbf{0 . 0 1 8}$ & $\mathbf{0 . 0 0 2}$ \\
\cline { 2 - 7 } & VCA & - & 1.768 & 0.182 & $\mathbf{0 . 0 1 8}$ & $\mathbf{0 . 0 0 2}$ \\
\hline \multirow{2}{*}{ N-FINDR } & N-FINDR & - & 2.113 & 0.530 & 0.254 & 0.466 \\
\cline { 2 - 7 } & ACO & - & 1.794 & 0.779 & 0.489 & 0.538 \\
\hline \multirow{3}{*}{ PPI } & PPI & - & - & 0.175 & $\mathbf{0 . 0 1 8}$ & $\mathbf{0 . 0 0 2}$ \\
\cline { 2 - 7 } & ACO & - & 1.702 & 0.180 & 0.033 & $\mathbf{0 . 0 0 2}$ \\
\hline \multirow{2}{*}{ VCA } & VCA & - & 1.759 & 0.175 & $\mathbf{0 . 0 1 8}$ & $\mathbf{0 . 0 0 2}$ \\
\cline { 2 - 7 } & ACO & - & 1.741 & $\mathbf{0 . 1 7 3}$ & $\mathbf{0 . 0 1 8}$ & $\mathbf{0 . 0 0 2}$ \\
\hline
\end{tabular}

The minimum values of spectral angle for each specific SNR value are outlined in bold typeface.

is increased gradually from a specified point, ranging within $[0,1]$ as simulated in [8]. To test the sensibility of the proposed multiple algorithm integration strategy to different noise levels, white Gaussian noise is added to the synthetic scene. The considered signal-to-noise ratios (SNRs) are 20:1, 40:1, 60:1, 80:1, and 100:1.

In order to study the impact of outliers on the considered endmember extraction algorithms, another image was simulated. It was generated by adding two outliers as shown in Fig. 6 into the previous simulated image with SNR $=100: 1$. More precisely, two outliers were located at spatial coordinates $(1,130)$ and $(130,200)$, with abundances $[0,0.85,0,0,-0.1]$ and $[0,0,0,1.1,0.1]$ corresponding to $r_{1}, r_{2}, r_{3}, r_{4}$, and $r_{5}$, respectively. In this figure, we plot the location of the outliers in a two-dimensional scatter plot given by the two principal components of the data.

\section{B. Results of Image Containing White Noise}

In our synthetic image experiments, the number of endmembers was set to five. To keep the balance between the computational complexity and accuracy, the synthetic image is subdivided into 16 subblocks in our proposed multiple algorithm integration strategy. This means that the two input parameters of our proposed approach are defined as follows: $p=5$ and $K=16$. As a result, four MNF components were used for the N-FINDR calculation, and five endmembers were 
TABLE II

RMSE BETWEen the ORIGINAL AND RECONSTRUCTED SCENE USING THE ENDMEMBERS EXTRACTED By Several Multiple Algorithm Integration Strategies

\begin{tabular}{c|c|c|c|c|c|c}
\hline Candidate endmembers & Endmember extraction & SNR = 20:1 & SNR = 40:1 & SNR = 60:1 & SNR $=\mathbf{8 0 : 1}$ & SNR $=\mathbf{1 0 0 : 1}$ \\
\hline \multirow{4}{*}{-} & N-FINDR & 0.1106 & 0.0120 & $\mathbf{0 . 0 0 1 2}$ & $1.24 \times 10^{-4}$ & $1.26 \times 10^{-5}$ \\
\cline { 2 - 7 } & PPI & 0.1656 & 0.1284 & $\mathbf{0 . 0 0 1 2}$ & $1.24 \times 10^{-4}$ & $1.26 \times 10^{-5}$ \\
\cline { 2 - 7 } & VCA & 0.1113 & 0.0122 & $\mathbf{0 . 0 0 1 2}$ & $1.24 \times 10^{-4}$ & $1.26 \times 10^{-5}$ \\
\hline \multirow{2}{*}{ N-FINDR } & N-FINDR & 0.1115 & 0.0125 & 0.0013 & $1.27 \times 10^{-4}$ & $1.60 \times 10^{-4}$ \\
\cline { 2 - 7 } & ACO & 0.1102 & $\mathbf{0 . 0 1 1 9}$ & 0.0013 & $1.77 \times 10^{-4}$ & $1.58 \times 10^{-4}$ \\
\hline \multirow{2}{*}{ PPI } & PPI & 0.1335 & 0.0422 & $\mathbf{0 . 0 0 1 2}$ & $1.24 \times 10^{-4}$ & $1.26 \times 10^{-5}$ \\
\cline { 2 - 7 } & ACO & 0.1106 & $\mathbf{0 . 0 1 1 9}$ & $\mathbf{0 . 0 0 1 2}$ & $\mathbf{1 . 2 1} \times \mathbf{1 0}^{-\mathbf{4}}$ & $\mathbf{1 . 2 0} \times \mathbf{1 0}^{-5}$ \\
\hline \multirow{2}{*}{ VCA } & VCA & 0.1105 & 0.0120 & $\mathbf{0 . 0 0 1 2}$ & $1.24 \times 10^{-4}$ & $1.26 \times 10^{-5}$ \\
\cline { 2 - 7 } & ACO & $\mathbf{0 . 1 0 9 3}$ & $\mathbf{0 . 0 1 1 9}$ & $\mathbf{0 . 0 0 1 2}$ & $1.23 \times 10^{-4}$ & $\mathbf{1 . 2 0} \times \mathbf{1 0}^{-5}$ \\
\hline
\end{tabular}

The minimum values of error for each specific SNR value are outlined in bold typeface.

TABLE III

Spectral Angle Scores (in Degrees) Between the Spectral in Fig. 4 And Their Corresponding Extracted ENDMEMBERS, AND THE RMSE BETWEEN ORIGINAL AND RECONSTRUCTED SCENE USING THE ENDMEMBERS EXTRACTED IN THE SYNTHETIC DATA (SNR $=100: 1)$ WITH OUTLIERS

\begin{tabular}{|c|c|c|c|c|c|c|c|c|}
\hline Candidate endmembers & Endmember extraction & $\mathbf{r}_{1}$ & $\mathbf{r}_{2}$ & $\mathbf{r}_{3}$ & $\mathbf{r}_{4}$ & $\mathbf{r}_{5}$ & Mean & RMSE \\
\hline \multirow{3}{*}{-} & N-FINDR & 0.001 & 0.002 & 0.002 & 0.485 & 0.004 & 0.099 & $7.11 \times 10^{-4}$ \\
\hline & PPI & 0.001 & 0.002 & 0.002 & 0.485 & - & - & 0.0186 \\
\hline & $\mathrm{VCA}$ & 0.001 & 2.102 & 0.002 & 0.001 & 0.004 & 0.422 & 0.0012 \\
\hline \multirow{2}{*}{ N-FINDR } & N-FINDR & 0.456 & 0.634 & 0.357 & 0.485 & 0.004 & 0.387 & $7.15 \times 10^{-4}$ \\
\hline & $\mathrm{ACO}$ & 0.456 & 0.634 & 0.357 & 0.089 & 0.004 & 0.308 & $1.72 \times 10^{-5}$ \\
\hline \multirow{2}{*}{ PPI } & PPI & 0.001 & 0.002 & 0.002 & 0.485 & - & - & 0.0186 \\
\hline & $\mathrm{ACO}$ & 0.001 & 0.002 & 0.002 & 0.039 & 0.004 & 0.009 & $1.26 \times 10^{-5}$ \\
\hline \multirow{2}{*}{$\mathrm{VCA}$} & VCA & 0.001 & 2.102 & 0.002 & 0.001 & 0.004 & 0.422 & 0.0012 \\
\hline & $\mathrm{ACO}$ & 0.001 & 0.002 & 0.002 & 0.001 & 0.003 & 0.002 & $1.24 \times 10^{-5}$ \\
\hline \multirow{6}{*}{-} & ICE & 1.916 & 2.955 & 1.353 & 1.452 & 3.068 & 2.149 & 0.0018 \\
\hline & MVC-NMF & - & 2.103 & 0.004 & 0.003 & 0.044 & - & $3.04 \times 10^{-4}$ \\
\hline & MVSA & 0.001 & - & 0.002 & 0.748 & 0.004 & - & $1.69 \times 10^{-5}$ \\
\hline & SISAL & 0.188 & 2.852 & 0.186 & 0.795 & - & - & $1.46 \times 10^{-5}$ \\
\hline & MVES & 0.001 & 6.836 & - & 6.014 & 5.097 & - & $1.56 \times 10^{-5}$ \\
\hline & RMVES & 3.021 & 4.710 & 3.551 & 5.559 & 5.734 & 4.515 & 0.0111 \\
\hline
\end{tabular}

The minimum values of error for each specific SNR value are outlined in bold typeface.

extracted from each subblock. Finally, five endmembers were extracted from 80 candidate endmembers by ACO.

Table I reports the endmember extraction results obtained after using different strategies. When only one endmember extraction algorithm is applied to the whole hyperspectral image, the column "candidate endmembers" in Table I is labeled as "-,", and the column "endmember extraction" shows the name of that algorithm. If our multiple algorithm integration strategy is used, the column "candidate endmembers" shows the name of the algorithm used for extracting candidate endmembers from subblocks, and the column "endmember extraction" shows the name of the algorithm used for extracting the final endmembers. It should also be noted that, when the spectral angle score [i.e., the angle (in degrees) between two spectral vectors] [24] of any endmember is higher than 10 degrees, we consider the extraction as a failure, and the mean value of the spectral angle scores for that particular strategy is simply labeled as "-." These conventions will be the same for Tables II-V.

Several simulated images with white noise $(\mathrm{SNRs}=20$ : $1,40: 1,60: 1,80: 1,100: 1$ ) are generated according to the LMM. Considering in the same noise level, the performances of several endmember extraction strategies do not differ from each other significantly as reported in Table I, since the simulated images are ideal according to LMM and pure pixel assumption. Namely, the proposed ACO-based integration strategy provides similar results as compared with the conventional methods, such as N-FINDR, PPI, and VCA in the ideal images. Then, we turn to focus on which method, among N-FINDR, PPI, and VCA, is more appropriate for 
TABLE IV

Spectral Angle Scores (in Degrees) Between USGS Mineral Spectra and Their Corresponding Endmembers EXtracted by Several Multiple Algorithm Integration Strategies From AVIRIS Cuprite Scene

\begin{tabular}{|c|c|c|c|c|c|c|c|}
\hline Preprocessing & Candidate endmembers & Endmember extraction & $\begin{array}{l}\text { Alunite } \\
\text { GDS84 }\end{array}$ & $\begin{array}{l}\text { Calcite } \\
\text { WS272 }\end{array}$ & $\begin{array}{c}\text { Kaolinite } \\
\text { KGa-1 }\end{array}$ & $\begin{array}{c}\text { Muscovite } \\
\text { GDS107 }\end{array}$ & Mean \\
\hline \multirow{9}{*}{-} & \multirow{3}{*}{-} & N-FINDR & 6.849 & - & - & - & - \\
\hline & & PPI & - & - & 6.763 & - & - \\
\hline & & VCA & 6.35 & 4.459 & 5.307 & - & - \\
\hline & \multirow{2}{*}{ N-FINDR } & N-FINDR & - & - & 4.821 & - & - \\
\hline & & $\mathrm{ACO}$ & 4.813 & - & 5.207 & - & - \\
\hline & \multirow{2}{*}{ PPI } & PPI & 6.350 & 6.115 & - & - & - \\
\hline & & $\mathrm{ACO}$ & 6.350 & 5.893 & 3.863 & 6.743 & 5.712 \\
\hline & \multirow{2}{*}{ VCA } & VCA & 4.186 & 4.459 & 6.134 & - & - \\
\hline & & $\mathrm{ACO}$ & 4.186 & 4.698 & 5.083 & 6.124 & 5.023 \\
\hline \multirow{6}{*}{ SSPP } & \multirow{3}{*}{-} & N-FINDR & 7.460 & - & - & 5.839 & - \\
\hline & & PPI & 4.133 & - & 4.243 & - & - \\
\hline & & VCA & 4.522 & 5.124 & - & 4.950 & - \\
\hline & N-FINDR & $\mathrm{ACO}$ & 7.481 & 4.905 & - & 5.245 & - \\
\hline & PPI & $\mathrm{ACO}$ & 4.531 & - & - & 4.530 & - \\
\hline & VCA & $\mathrm{ACO}$ & 4.891 & 4.905 & 4.243 & 4.390 & 4.607 \\
\hline \multirow{6}{*}{-} & \multirow{6}{*}{-} & ICE & 6.385 & 6.277 & 5.324 & 5.291 & 5.819 \\
\hline & & MVC-NMF & 6.450 & 6.104 & 5.295 & 4.755 & 5.651 \\
\hline & & MVSA & - & 6.887 & 7.709 & 8.518 & - \\
\hline & & SISAL & - & 5.772 & - & - & - \\
\hline & & MVES & - & 7.346 & 7.539 & 7.832 & - \\
\hline & & RMVES & - & 4.570 & 7.558 & 5.316 & - \\
\hline
\end{tabular}

The minimum values for four typical minerals in the cuprite mining district are outlined in bold typeface.

the candidate endmember extraction in ACO-based integration strategy. Comparing the results of "N-FINDR + ACO," "PPI + ACO," and "VCA + ACO" in Table I, the "VCA + ACO" perform the best in most cases and show higher stability in different noise conditions, whereas the "N-FINDR + ACO" perform the worst (even worse than the single N-FINDR). This means that N-FINDR is not suitable for being applied in the subimages, when the number of subimage endmembers is set to be as many as the global image. Hence the VCA is suggested as a better choice to extract candidate endmember for ACO-based integration strategy.

Table II reports the RMSEs achieved after reconstructing the synthetic scenes using the endmembers extracted by several multiple algorithm integration strategies. When the SNR is very low (such as 20:1), the provided endmembers cannot be used to reconstruct the original hyperspectral image with low RMSE. On the other hand, if the SNR is higher than 40:1, the results provided by the strategies based on ACO exhibit very low reconstruction errors. Overall, the results reported on Table II are in very good agreement with those previously reported in Table I using the spectral angle distance as an evaluation metric. For instance, Table II also reveals that N-FINDR is not suitable for the proposed multiple algorithm integration strategy. On the other hand, ACO provides very good results for the final endmember extraction, improving significantly the results obtained by PPI and VCA. The "VCA + ACO" strategy consistently provides the best results across almost all the considered hyperspectral scenes with different SNRs.

\section{Results in Cases With Outliers}

The hyperspectral images that need to be processed in practice are usually not as perfect as the ones that we have considered under a perfect LMM assumption. The influence of outliers for endmember extraction was also tested in an experiment with outliers. The corresponding results are shown in Table III and in Fig. 7. Although the considered SNR is quite high (100:1), PPI, N-FINDR, and VCA algorithms all mistakenly consider at least one outlier as an endmember, which can be observed clearly in Fig. 7. As a result, some of the average spectral angles of these three algorithms are quite large. Moreover, the results of "N-FINDR + N-FINDR," "PPI + PPI," and "VCA + VCA" almost follow a similar pattern to N-FINDR, PPI, and VCA, which means that the simple data partitioning seems not to make any contribution for the already established endmember extraction algorithms. The ACO cannot extract correct endmembers from the candidate endmembers provided by N-FINDR. This experiment 
TABLE V

ERror SCORES AFTER RECONSTRUCTING THE AVIRIS CUPRITE SCENE USING THE ENDMEMBERS EXTRACTED By SEVERAL Multiple Algorithm Integration Strategies

\begin{tabular}{|c|c|c|c|}
\hline Preprocessing & $\begin{array}{c}\text { Candidate } \\
\text { endmembers }\end{array}$ & $\begin{array}{c}\text { Endmember } \\
\text { extraction }\end{array}$ & RMSE \\
\hline \multirow{9}{*}{-} & \multirow{3}{*}{ - } & N-FINDR & 4.480 \\
\hline & & PPI & 5.902 \\
\hline & & $\mathrm{VCA}$ & 4.267 \\
\hline & \multirow{2}{*}{ N-FINDR } & N-FINDR & 4.783 \\
\hline & & $\mathrm{ACO}$ & 3.564 \\
\hline & \multirow{2}{*}{ PPI } & PPI & 8.861 \\
\hline & & $\mathrm{ACO}$ & 3.487 \\
\hline & \multirow{2}{*}{ VCA } & $\mathrm{VCA}$ & 4.269 \\
\hline & & $\mathrm{ACO}$ & 3.326 \\
\hline \multirow{6}{*}{ SSPP } & \multirow{3}{*}{ - } & N-FINDR & 5.061 \\
\hline & & PPI & 4.951 \\
\hline & & VCA & 3.846 \\
\hline & N-FINDR & $\mathrm{ACO}$ & 3.996 \\
\hline & PPI & $\mathrm{ACO}$ & 3.287 \\
\hline & $\mathrm{VCA}$ & $\mathrm{ACO}$ & 3.241 \\
\hline \multirow{7}{*}{-} & \multirow{7}{*}{-} & ICE & 3.492 \\
\hline & & MVC-NMF & 2.953 \\
\hline & & MVSA & 2.585 \\
\hline & & SISAL & 2.589 \\
\hline & & MVES & 2.587 \\
\hline & & RMVES & 3.524 \\
\hline & & $\begin{array}{c}\text { Sparse } \\
\text { Unmixing }\end{array}$ & 6.327 \\
\hline
\end{tabular}

The minimum value is outlined in bold typeface.

confirms our introspection that N-FINDR is not appropriate for extracting candidate endmembers in our considered multiple algorithm integration strategy. However, the combinations "PPI + ACO" or "VCA + ACO" provide results which are largely improved.

As mentioned above, N-FINDR, PPI, and VCA are extremely sensitive to outliers. It may be unfair to compare the proposed method with these three algorithms only, since the field of endmember extraction algorithms has developed rapidly in recent years. Consequently, it requires a comparison with the state-of-the-art endmember extraction algorithms, such as ICE [46], MVC-NMF [13], MVSA [12], SISAL [11], MVES [14], RMVES [47], and sparse unmixing method.

Importantly, recent developments in the literature [2], [11] [12], [47] reveal that, among them, ICE, MVSA, SISAL, and RMVES are the ones providing high robustness to outliers or noise. To be precise, ICE can be robust in the sense of allowing data points to be outside of the simplex volume, since it uses a regularization parameter $\mu$ to control the tradeoff between RMSE and smaller simplexes. On the other hand, MVSA and SISAL implement a robust version of the minimum volume concept by allowing the positivity constraint to be violated. The RMVES, a robust version of MVES, can account for the noise effects in the observations by employing soft constraints on the fractional abundances, which control the volume of the resulting simplex. Overall, the MVSA, SISAL, and RMVES bring robustness to the extraction problem by allowing violations of the ANC.

In addition, note that the original versions of the above robust algorithms were used instead of the two-tiered versions in all our experiments. Because we have conducted the experiments related to the two-tiered robust algorithms previously, namely we made them find the final set of endmembers using the patchbased approach, just as the ACO-based strategy did. The results show that the performance of patch-based robust algorithms did not change a lot compared with the original versions in the synthetic data and cuprite data. Actually, the main improvement of our approach is the mechanism introduced by ACO, not the partitioning operation. The robust algorithms and established methods (N-FINDR, PPI, and VCA) did not benefit from the simple partitioning. So, for space considerations, all our experiments only report the results obtained with the original versions of the aforementioned robust algorithms.

In our simulation experiments, we did not conduct the comparison with sparse unmixing method due to the fact that the synthetic data are generated using the library spectra. It would be the same spectral library where the sparse unmixing selects endmembers from. In this case, sparse unmixing would certainly get a very good performance. However, using library endmembers, rarely acquired under the same conditions as the airborne data, is believed to be a potential drawback of sparse unmixing and to result in the risk of unmixing error. So, instead of comparing with sparse unmixing in the simulation experiments, we conducted the comparison in the cuprite data.

With these considerations in mind, the state-of-the-art methods, i.e., ICE, MVC-NMF, MVSA, SISAL, MVES, and RMVES, have been compared with the proposed strategies in the presence of outliers. As reported in Table III and shown in Fig. 8, only ICE and RMVES can extract all the five endmembers, however, with higher RMSE, whereas the MVC-NMF, MVSA, SISAL, and MVES, coming with lower RMSE, did not extract all the endmembers successfully. The above-mentioned results reveal two kinds of performances, despite they all belong to the minimum volume-based algorithms [2]. It is mainly caused by the regularization parameters, which make a tradeoff between the RMSE and minimum simplex volume and need to be hand tuned. In our experiments, excluding ICE, the regularization parameters were set to default values suggested by the authors. It was pointed out that the solution of ICE can be sensitive to the choice of the regularization parameter [2], so we adjusted it several times intending to get the best outcome. In fact, reaching a compromise between lower RMSE and minimum simplex volume is of critical importance for minimum volume-based algorithms. For instance, ICE allows data points (sometimes it may be outliers or noise points) to be outside of the simplex volume, which results in higher RMSE as well as smaller simplex volume. In this way, ICE brings robustness to endmember extraction problem. As reported in Table III, ICE performs best among these six algorithms in the 

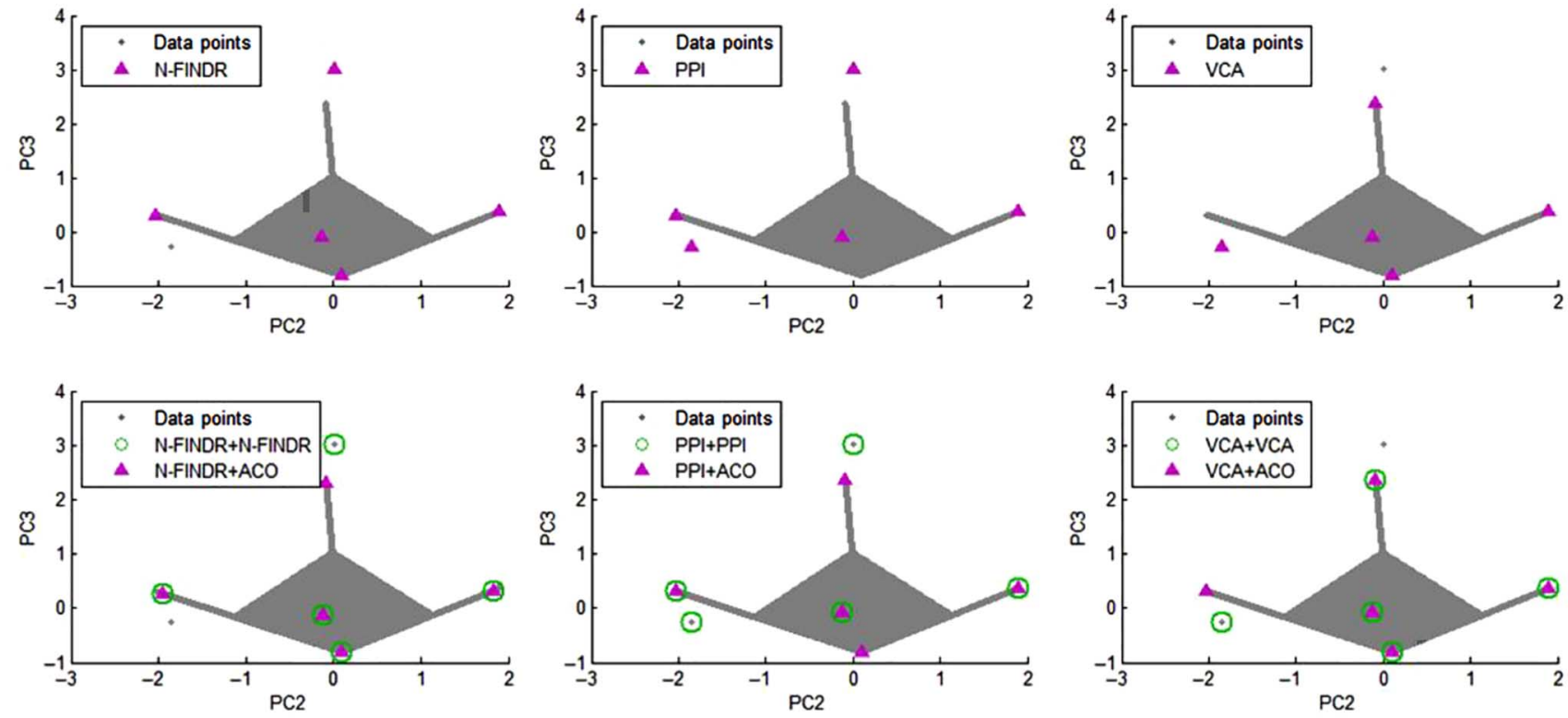

Fig. 7. Endmembers extracted from the synthetic data with outliers using different combinations for the multiple algorithm integration strategy.

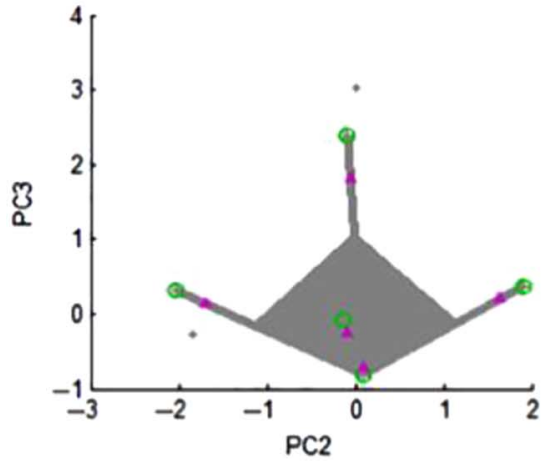

(a)

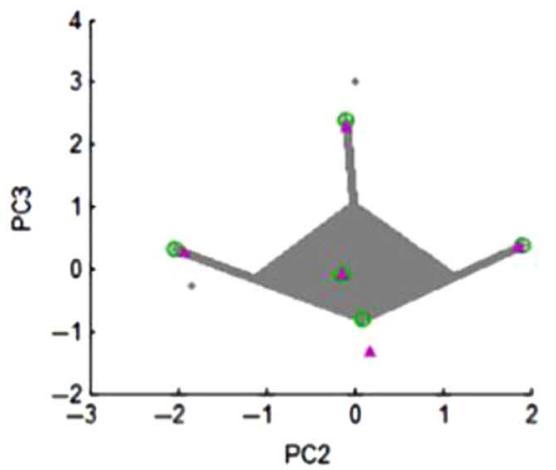

(d)

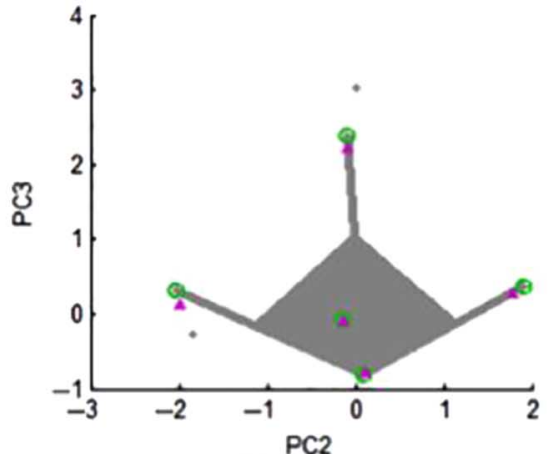

(b)

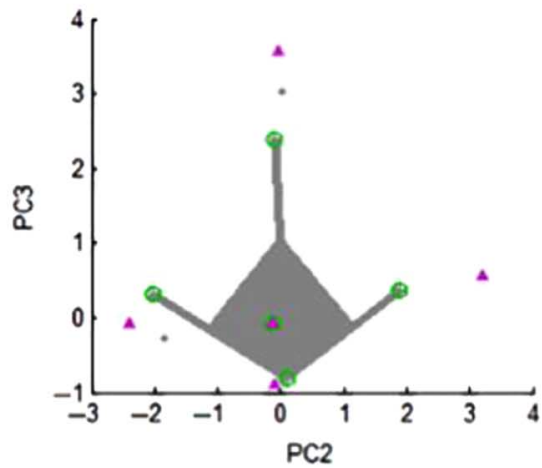

(e)

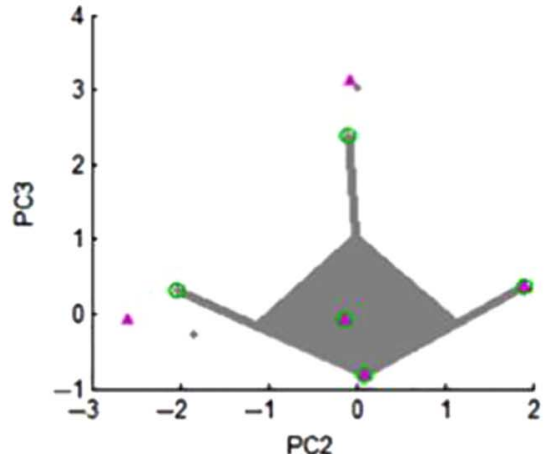

(c)

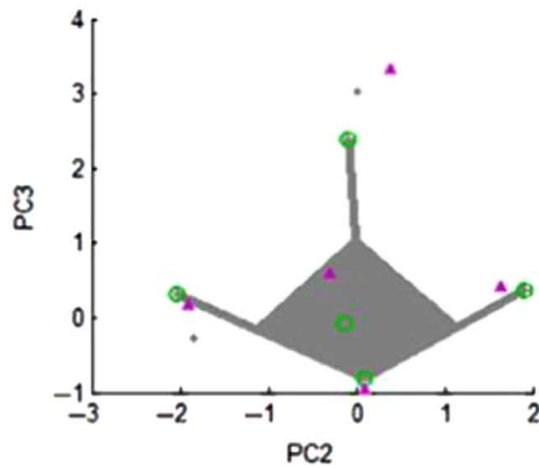

(f)

\section{- Data points 0 True endmembers $\Delta$ Estimated endmembers}

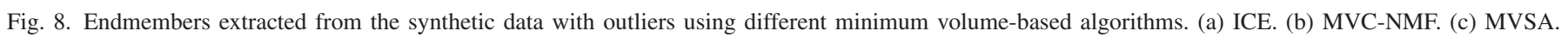
(d) SISAL. (e) MVES. (f) RMVES.

simulation experiments. However, its extraction accuracy is still not as good as the proposed ACO-based methods. The influence of outliers can be illustrated more clearly in the scatter plots (Fig. 8). Obviously, all these six algorithms are influenced by such kind of outliers more or less, and some of the estimated endmembers deviate from the true endmembers.
Therefore, it can be concluded that the proposed ACO-based method can well treat complex situations with or without outliers. The performance of ACO-based method is significantly better than other algorithms when they face a scenario with outliers, as indicated in the experiments conducted in this section. 


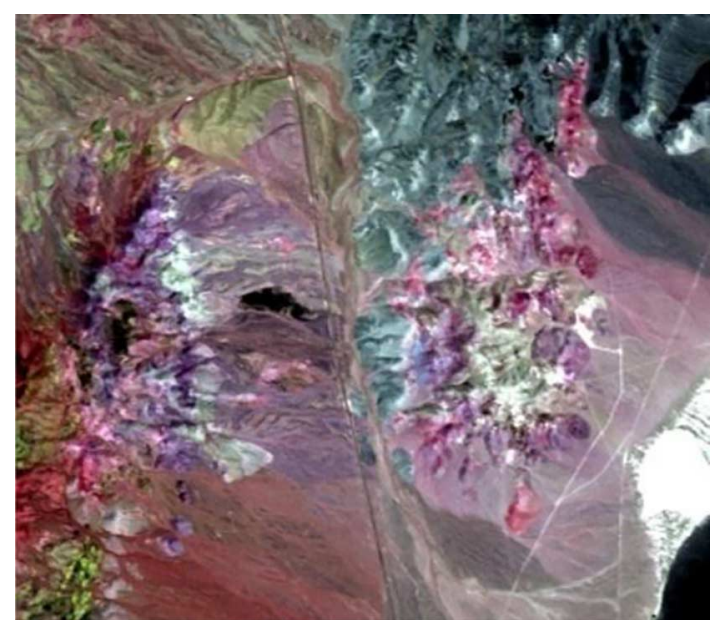

Fig. 9. False color composition of AVIRIS hyperspectral image at cuprite, Nevada.

\section{EXPERIMENTS With REAL DATA}

\section{A. Real Data and Experimental Design}

Our experimental results with real data are based on the cuprite scene acquired by AVIRIS [48]. The scene is illustrated in Fig. 9. It has been commonly adopted for evaluating the performance of spectral unmixing algorithms. Several minerals of interest, such as alunite, calcite, kaolinite, and muscovite, can be found in this mining district [49]. The cuprite image used in this section comprises $400 \times 350$ pixels in size, and 50 spectral bands ranging from 1.99 to $2.48 \mu \mathrm{m}$.

In this experiment, the number of endmembers in the cuprite image is first estimated by HySime, which provided an estimation of $p=8$ endmembers. The cuprite scene is subdivided into $K=10$ subblocks, with eight endmembers extracted from each subblock.

In real hyperspectral images, the spatial relationships between pixels should not be ignored. In order to account for spatial information in our analysis, we conducted a preprocessing of the scene using SSPP method [24]. Since the input image is not regular after applying the SSPP, the strategy cannot use subdivided subblocks to provide candidate endmembers for ACO. As a result, in this case, we use random selection to generate subsets. This means that the subset generation is simply conducted by randomly selecting candidate pixels provided by SSPP.

\section{B. Experimental Results and Analysis}

Table IV reports the spectral angle scores between some selected United States Geological Survey (USGS) mineral spectra and their corresponding endmembers extracted by several multiple algorithm integration strategies. If the column "candidate endmembers" is labeled as "-" in the strategy with SSPP, this means that only the algorithm shown in the "endmember extraction" column is used to directly extract final endmembers from the candidate pixels provided by SSPP. Otherwise, we assume that the final endmembers are extracted

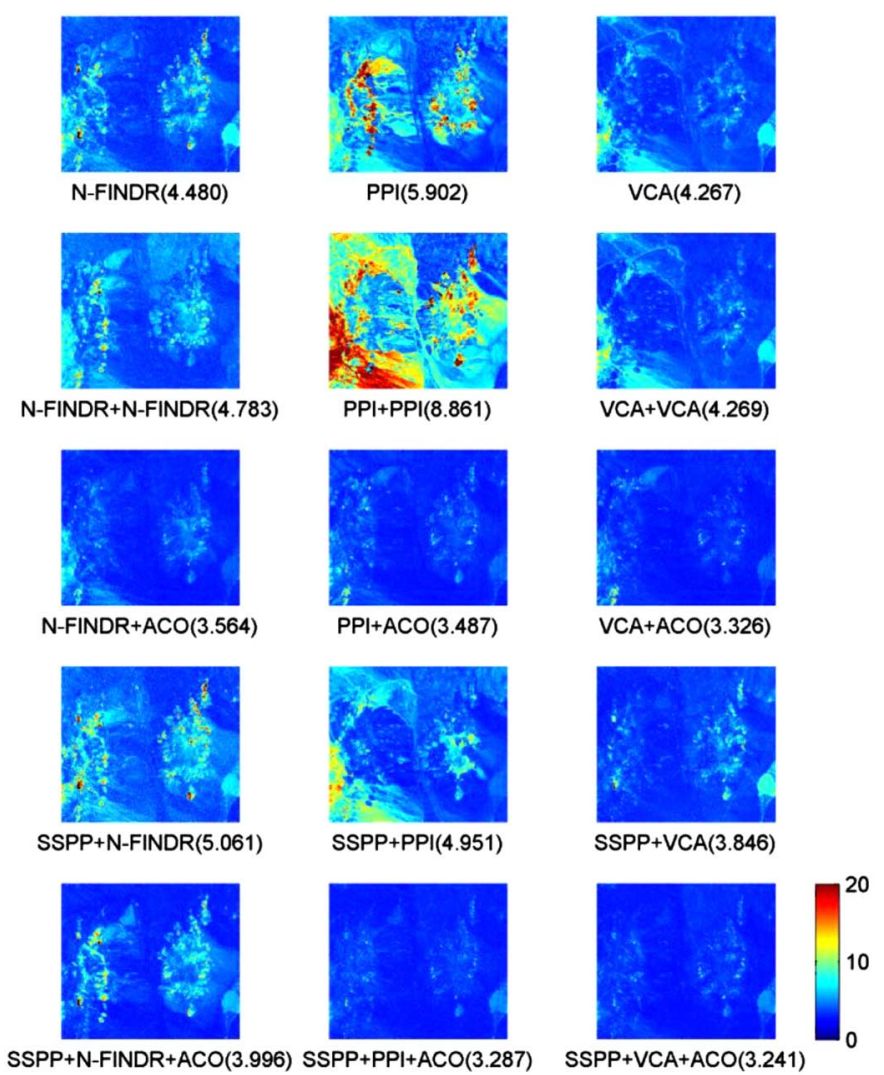

Fig. 10. Errors measured for various endmember extraction strategies after reconstructing the AVIRIS cuprite hyperspectral image scene.

by ACO after SSPP preprocessing and candidate endmember extraction.

At this point, it is important to emphasize that only four endmembers are reported in the tables in order to provide a comparable result between the different algorithms. The compared methods may extract different minerals since the cuprite contains abundant mineral materials. So, only four highly representative minerals (i.e., alunite, calcite, kaolinite, and muscovite, which can be extracted by all the compared methods) in the cuprite mining district are used in this work to substantiate endmember signature purity.

In the pure pixel-based methods, only the "PPI + ACO," "VCA + ACO," and "SSPP + VCA + ACO" have successfully extracted all the four considered endmembers as reported in Table IV. Other strategies failed may due to insufficient spectral information provided by the 50 available spectral bands. The successful strategies imply that the proposed ACO-based approach can improve endmember extraction in the considered real scene. Note that "N-FINDR + ACO" does not perform as effectively as other ACO-based methods. A similar observation also happened in the synthetic data experiments, and the reasons were analyzed before. On the other hand, the "SSPP + VCA + ACO" provides the best endmember extraction performance. However, other strategies do not benefit from the SSPP-based preprocessing. The spatial information may not always improve endmember extraction, so for our proposed ACO-based strategy we think that SSPP-based preprocessing may be considered as an optional, but not necessary step. 
TABLE VI

Processing Time (in Seconds) Measured in a Desktop PC with an Intel Xeon X5660 CPU, 12 Gb Ram, AND AN NVIDIA QUADRO 5000 GPU

\begin{tabular}{c|c|c|c|c}
\hline Algorithms & Preprocessing time & $\begin{array}{c}\text { Candidate endmember } \\
\text { extraction time }\end{array}$ & $\begin{array}{c}\text { Final endmember } \\
\text { extraction time }\end{array}$ & Total processing time \\
\hline $\mathrm{VCA}$ & - & - & 0.46 & 0.46 \\
\hline $\mathrm{VCA}+\mathrm{VCA}$ & - & 0.80 & 0.03 & 0.83 \\
\hline $\mathrm{VCA}+\mathrm{ACO}$ & - & 0.80 & 748.57 & 749.37 \\
\hline $\mathrm{SSPP}+\mathrm{VCA}$ & 15.16 & - & 0.05 & 15.21 \\
\hline $\mathrm{SSPP}+\mathrm{VCA}+\mathrm{ACO}$ & 15.16 & 0.59 & 747.98 & 763.73 \\
\hline
\end{tabular}

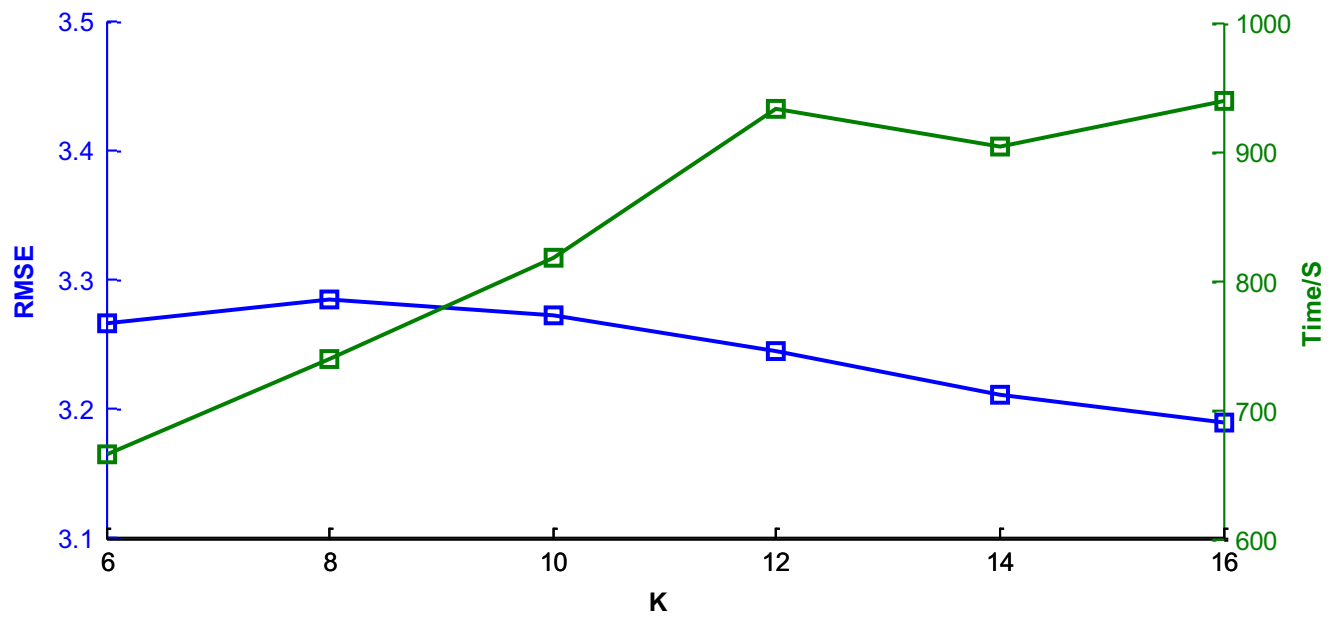

Fig. 11. Performance of "VCA + ACO" method when the number of subblocks increased, where the approach is run 10 times (the average results are reported).

Moreover, the performance of the proposed approach is also compared to six state-of-the-art minimum volume-based algorithms and sparse unmixing approach. Importantly, among them, ICE, MVSA, SISAL, and RMVES are reported in recent developments as being able to provide robustness to outliers or noise. In our experiments with cuprite data, only ICE and MVC-NMF can extract all the four considered endmembers, as reported in Table IV. However, the mean spectral angle scores of "VCA + ACO" and "SSPP + VCA + ACO" are better than that of ICE and MVC-NMF. Note that, due to the fact that sparse unmixing only selects endmembers from a spectral library, our comparison of spectral angle scores between USGS mineral spectra and their corresponding extracted endmembers does not include sparse unmixing.

The RMSEs after reconstructing the AVIRIS cuprite scene using the endmember extracted by different methods are also reported in Table V. The ACO-based methods obtained relatively lower RMSEs than other pure pixel-based methods since the objective function of $\mathrm{ACO}$ is focused on minimizing the RMSE. Note that the "SSPP + VCA + ACO" obtained the smallest RMSE as well as the smallest spectral angle scores in the pure pixel-based methods. This means that it is reasonable to use minimum RMSE as the objective function for ACO.

The error maps obtained after reconstructing the AVIRIS cuprite hyperspectral image scene using different multiple integration strategies are shown as Fig. 10. The "N-FINDR + ACO" obtains the best error maps in all results obtained using N-FINDR in any part of the chain. On the other hand, the result obtained by "SSPP + PPI + ACO" is better than the one obtained "SSPP + PPI." The experiments with VCA show similar behavior, with "VCA + ACO" and "SSPP + VCA + ACO," respectively, providing the best results in each row of Fig. 10. The results indicate that the proposed multiple algorithm integration strategy based on ACO is an effective method for endmember extraction.

The smaller RMSE can lead to better endmember extraction performance in the pure pixel-based methods, whereas it may fail in the minimum volume-based methods. The ICE, MVCNMF, MVSA, SISAL, MVES, and RMVES obtain smaller RMSEs than the pure pixel-based methods as reported in Table V. In fact, these algorithms do not all extract the considered endmembers according to the spectral angle scores. On the other hand, the RMSE of sparse unmixing is much higher than that of "VCA + ACO" or "SSPP + VCA + ACO."

As we have mentioned before, the ACO strategy used for endmember extraction is time consuming so that the candidate endmembers extraction step is quite necessary. Here, we take strategies combining with VCA method as an example and report their processing times (in seconds) in Table VI. The computer utilized in the experiments is equipped with an Intel Xeon X5660 CPU, 12 GB RAM and an NVidia Quadro 5000 GPU. As shown by Table VI, the total processing times are increased but the ACO strategy is highly parallel and can be effectively implemented using HPC architectures.

\section{Adjustable Parameter Discussion}

To conclude our experimental assessment, we provide a discussion on the adjustable parameters of the proposed method. There are two parameters, the number of endmembers $p$ and the 
number of subblocks $K$, required by the ACO-based algorithm. The value of $p$ is suggested to be estimated by HySime algorithm, while the value of $K$ needs to be hand-tuned. Different values of $K$ may introduce a distinct effect on the performance in terms of the RMSE and computing time, as depicted in Fig. 11. We emphasize that our implementation was carried out using MATLAB R2010 on a desktop PC equipped with an Intel Xeon X5660 CPU, 12 GB RAM, and an NVidia Quadro 5000 GPU. As shown in Fig. 11, the method has a slightly lower RMSE when the number of subblocks is increased. More subblocks would result in sufficient candidate endmembers as well as higher computing time. As a result, in our experiments with the cuprite image, $K$ is set to ten in order to reduce the computational complexity.

\section{CONCLUSION AND DisCUSSION}

In this paper, we have developed a new multiple integration strategy for endmember extraction based on ACO. Our experimental results, conducted using several classic endmember extraction algorithms, indicate that the proposed ACO-based strategy can provide a set of endmembers which are more useful for unmixing purposes than those obtained by the individual methods. Moreover, the experiment results clearly show that what makes sense to the improvement of our approach is the mechanism introduced by $\mathrm{ACO}$, not the partitioning operation. Besides, when comparing our approach with state-of-the-art algorithms, we have found that the proposed ACO-based framework shows more robustness against noise and outliers. As a result, a main strength of the proposed approach is its capacity to automatically find the best possible combination out of a set of candidate endmembers generated using different methods, as well as its capacity to deal with noise and outliers. Our proposed strategy has been experimentally validated using both simulated scenes and a real hyperspectral scene collected by the AVIRIS over the cuprite mining district in Nevada. The results obtained indicate that the proposed multiple algorithm integration approach can provide optimal solutions with higher spectral purity, better fractional abundance estimation, and lower reconstruction errors than those provided using the considered classic strategies.

As with any new approach, there are some unresolved issues that may present challenges over time. For instance, an aspect to be addressed in future developments is the computational complexity of the proposed approach, which can be effectively tackled by taking advantage of the inherently parallel nature of the ACO strategy, which maps well in many different types of HPC architectures. Another important topic deserving future research is how to combine the results provided by different endmember extraction algorithms designed under different principles, e.g., algorithms with and without the pure pixel assumption, or algorithms with and without spatial constraints. This aspect represents an important future development that may help combine the results provided by the plethora of available techniques for endmember extraction. Although our experimental results are constrained to three popular algorithms: PPI, N-FINDR, and VCA (selected for the purpose of illustrating our concept), additional results should be explored in future developments by combining more diverse strategies for endmember extraction.

\section{ACKNOWLEDGMENT}

The authors acknowledge the Jet Propulsion Laboratory for generously providing the AVIRIS data. They also acknowledge the anonymous reviewers for their outstanding comments and suggestions, which greatly helped to improve the technical content and presentation of the manuscript.

\section{REFERENCES}

[1] N. Keshava and J. F. Mustard, "Spectral unmixing," IEEE Signal Process. Mag., vol. 19, no. 1, pp. 44-57, Aug. 2002.

[2] J. Bioucas-Dias et al., "Hyperspectral unmixing overview: Geometrical, statistical, and sparse regression-based approaches," IEEE J. Sel. Topics Appl. Earth Observ. Remote Sens., vol. 5, no. 2, pp. 354-379, Apr. 2012.

[3] C. Quintano, A. Fernández-Manso, Y. E. Shimabukuro, and G. Pereira, "Spectral unmixing," Int. J. Remote Sens., vol. 33, no. 17, pp. 5307-5340, 2012.

[4] B. Hapke, "Bidirectional reflectance spectroscopy. I. Theory," $J$. Geophys. Res., vol. 86, pp. 3039-3054, 1981.

[5] Y. Shkuratov, L. Starukhina, H. Hoffmann, and G. Arnold, "A model of spectral albedo of particulate surfaces: Implications for optical properties of the Moon," Icarus, vol. 137, pp. 235-246, 1999.

[6] S. Jacquemoud and F. Baret, "PROSPECT: A model of leaf optical properties spectra," Remote Sens. Environ., vol. 34, no. 2, pp. 75-91, 1990.

[7] W. Verhoef, "Light scattering by leaf layers with application to canopy reflectance modeling: The SAIL model," Remote Sens. Environ., vol. 16, no. 2, pp. 125-141, 1984.

[8] A. Plaza, P. Martínez, R. Pérez, and J. Plaza, "A quantitative and comparative analysis of endmember extraction algorithms from hyperspectral data," IEEE Trans. Geosci. Remote Sens., vol. 42, no. 3, pp. 650-663, Mar. 2004.

[9] J. M. P. Nascimento and J. Bioucas-Dias, "Does independent component analysis play a role in unmixing hyperspectral data?" IEEE Trans. Geosci. Remote Sens., vol. 43, no. 1, pp. 175-187, Jan. 2005.

[10] N. Dobigeon, S. Moussaoui, M. Coulon, J.-Y. Tourneret, and A. O. Hero, "Joint Bayesian endmember extraction and linear unmixing for hyperspectral imagery," IEEE Trans. Signal Process., vol. 57, no. 11, pp. 43554368, Oct. 2009.

[11] J. Bioucas-Dias. "A variable splitting augmented Lagrangian approach to linear spectral unmixing," in Proc. IEEE 1st Workshop Hyperspectral Image Signal Process. Evol. Remote Sens., 2009, pp. 1-4.

[12] J. Li and J. Bioucas-Dias, "Minimum volume simplex analysis: A fast algorithm to unmix hyperspectral data," in Proc. IEEE Int. Geosci. Remote Sens. Symp. (IGARSS), 2008, vol. 3, pp. 250-253.

[13] L. Miao and H. Qi, "Endmember extraction from highly mixed data using minimum volume constrained nonnegative matrix factorization," IEEE Trans. Geosci. Remote Sens., vol. 45, no. 3, pp. 765-777, Feb. 2007.

[14] T. H. Chan, C. Y. Chi, Y. M. Huang, and W. K. Ma, "A convex analysis based minimum-volume enclosing simplex algorithm for hyperspectral unmixing," IEEE Trans. Signal Process., vol. 57, no. 11, pp. 4418-4432, Jun. 2009.

[15] A. Ifarraguerri and C.-I. Chang, "Multispectral and hyperspectral image analysis with convex cones," IEEE Trans. Geosci. Remote Sens., vol. 37 , no. 2, pp. 756-770, Mar. 1999.

[16] J. W. Boardman and F. A. Kruse, "Analysis of imaging spectrometer data using N-dimensional geometry and a mixture-tuned matched filtering approach," IEEE Trans. Geosci. Remote Sens., vol. 49, no. 11, pp. 4138-4152, Aug. 2011.

[17] M. E. Winter, "A proof of the N-FINDR algorithm for the automated detection of endmembers in a hyperspectral image," in Proc. SPIE, 2004, vol. 5425, pp. 31-41.

[18] R. A. Neville, K. Staenz, T. Szeredi, J. Lefebvre, and P. Hauff, "Automatic endmember extraction from hyperspectral data for mineral exploration," in Proc. 21st Can. Symp. Remote Sens., 1999, pp. 21-24.

[19] J. M. P. Nascimento and J. Bioucas-Dias, "Vertex component analysis: A fast algorithm to unmix hyperspectral data," IEEE Trans. Geosci. Remote Sens., vol. 43, no. 4, pp. 898-910, Apr. 2005. 
[20] C. I. Chang, C. C. Wu, W. M. Liu, and Y. C. Ouyang, "A new growing method for simplex-based endmember extraction algorithm," IEEE Trans. Geosci. Remote Sens., vol. 44, no. 10, pp. 2804-2819, Sep. 2006.

[21] J. Gruninger, A. J. Ratkowski, and M. L. Hoke, "The sequential maximum angle convex cone (SMACC) endmember model," in Proc. SPIE, 2004, vol. 5425, pp. 1-14.

[22] A. Plaza, P. Martinez, R. Perez, and J. Plaza, "Spatial/spectral endmember extraction by multidimensional morphological operations," IEEE Trans. Geosci. Remote Sens., vol. 40, no. 9, pp. 2025-2041, Dec. 2002.

[23] D. M. Rogge et al., "Integration of spatial-spectral information for the improved extraction of endmembers," Remote Sens. Environ., vol. 110, no. 3, pp. 287-303, 2007.

[24] G. Martín and A. Plaza. "Spatial-spectral preprocessing prior to endmember identification and unmixing of remotely sensed hyperspectral data," IEEE J. Sel. Topics Appl. Earth Observ. Remote Sens., vol. 5, no. 2, pp. 380-395, Apr. 2012.

[25] M. D. Iordache, J. Bioucas-Dias, and A. Plaza, "Sparse unmixing of hyperspectral data," IEEE Trans. Geosci. Remote Sens., vol. 49, no. 6, pp. 2014-2039, Jan. 2011.

[26] M. D. Iordache, J. Bioucas-Dias, and A. Plaza, "Total variation spatial regularization for sparse hyperspectral unmixing," IEEE Trans. Geosci. Remote Sens., vol. 50, no. 11, pp. 4484-4502, May 2012.

[27] Y. F. Zhong, R. Y. Feng, and L. P. Zhang, "Non-local sparse unmixing for hyperspectral remote sensing imagery," IEEE J. Sel. Topics Appl. Earth Observ. Remote Sens., vol. 7, no. 6, pp. 1889-1909, Jun. 2014.

[28] B. Somers, M. Zortea, A. Plaza, and G. P. Asner, "Automated extraction of image-based endmember bundles for improved spectral unmixing," IEEE J. Sel. Topics Appl. Earth Observ. Remote Sens., vol. 5, no. 2, pp. 396-408, Jan. 2012.

[29] B. Zhang, X. Sun, L. R. Gao, and L. N. Yang, "Endmember extraction of hyperspectral remote sensing images based on the ant colony optimization (ACO) algorithm," IEEE Trans. Geosci. Remote Sens., vol. 49, no. 7, pp. 2635-2646, Mar. 2011.

[30] B. Zhang, J. W. Gao, L. R. Gao, and X. Sun, "Improvements in the ant colony optimization algorithm for endmember extraction from hyperspectral images," IEEE J. Sel. Topics Appl. Earth Observ. Remote Sens., vol. 6, no. 2, pp. 522-530, Jan. 2013.

[31] J. M. Bioucas-Dias and J. M. P. Nascimento, "Hyperspectral subspace identification," IEEE Trans. Geosci. Remote Sens., vol. 46, no. 8, pp. 2435-2445, Aug. 2008.

[32] A. A. Green, M. Berman, P. Switzer, and M. D. Craig, "A transformation for ordering multispectral data in terms of image quality with implications for noise removal," IEEE Trans. Geosci. Remote Sens., vol. 26, no. 1, pp. 65-74, Jan. 1988.

[33] A. Plaza, D. Valencia, J. Plaza, and P. Martinez, "Commodity clusterbased parallel processing of hyperspectral imagery," J. Parallel Distrib. Comput., vol. 66, no. 3, pp. 345-358, 2006.

[34] A. Plaza, J. Plaza, A. Paz, and S. Sánchez, "Parallel hyperspectral image and signal processing," IEEE Signal Process. Mag., vol. 28, no. 3, pp. 119-126, May 2011

[35] A. Barberis, G. Danese, F. Leporati, A. Plaza, and E. Torti, "Real-time implementation of the vertex component analysis algorithm on GPUs," IEEE Geosci. Remote Sens. Lett., vol. 10, no. 2, pp. 251-255, Mar. 2013.

[36] A. Colorni, M. Dorigo, and V. Maniezzo, "Distributed optimization by ant colonies," in Proc. ECAL91-Eur. Conf. Artif. Life, 1991, pp. 134-142.

[37] M. Dorigo, "Optimization, learning and natural algorithms," Ph.D. dissertation, Dipartimento di Elettronica, Politecnico di Milano, Milano, Italy, 1992.

[38] Y. F. Zhong, L. Zhao, and L. P. Zhang, "An adaptive differential evolution endmember extraction algorithm for hyperspectral remote sensing imagery," IEEE Geosci. Remote Sens. Lett., vol. 11, no. 6, pp. 1061-1065, Nov. 2013.

[39] D. W. J. Stein et al., "Anomaly detection from hyperspectral imagery," IEEE Signal Process. Mag., vol. 19, no. 1, pp. 58-69, Aug. 2002.

[40] S. Kumar, J. Ghosh, and M. M. Crawford, "Hierarchical fusion of multiple classifiers for hyperspectral data analysis," Pattern Anal. Appl., vol. 5, no. 2, pp. 210-220, 2002.

[41] J. M. Liu and J. S. Zhang, "A new maximum simplex volume method based on householder transformation for endmember extraction," IEEE Trans. Geosci. Remote Sens., vol. 50, no. 1, pp. 104-118, Dec. 2011.

[42] A. Plaza, Q. Du, Y. L. Chang, and R. L. King, "High performance computing for hyperspectral remote sensing," IEEE J. Sel. Topics Appl. Earth Observ. Remote Sens., vol. 4, no. 3, pp. 528-544, Sep. 2011.

[43] M. Zortea and A. Plaza, "A quantitative and comparative analysis of different implementations of N-FINDR: A fast endmember extraction algorithm," IEEE Geosci. Remote Sens. Lett., vol. 6, no. 4, pp. 787-791, Oct. 2009.
[44] A. Plaza, D. Valencia, J. Plaza, and C. I. Chang, "Parallel implementation of endmember extraction algorithms from hyperspectral data," IEEE Geosci. Remote Sens. Lett., vol. 3, no. 3, pp. 334-338, Jul. 2006.

[45] W. F. Luo, B. Zhang, and X. P. Jia, "New improvements in parallel implementation of N-FINDR algorithm," IEEE Trans. Geosci. Remote Sens., vol. 50, no. 10, pp. 3648-3659, Sep. 2012.

[46] M. Berman et al., "ICE: A statistical approach to identifying endmembers in hyperspectral images: Learning from earth's shapes and colors," IEEE Trans. Geosci. Remote Sens., vol. 42, no. 10, pp. 2085-2095, Oct. 2004.

[47] A. Ambikapathi, T. H. Chan, W. K. Ma, and C. Y. Chi. "Chanceconstrained robust minimum-volume enclosing simplex algorithm for hyperspectral unmixing," IEEE Trans. Geosci. Remote Sens., vol. 49, no. 11, pp. 4194-4209, Oct. 2011.

[48] R. O. Green et al., "Imaging spectroscopy and the Airborne Visible/Infrared Imaging Spectrometer (AVIRIS)," Remote Sens. Environ., vol. 65, no. 3, pp. 227-248, 1998.

[49] M. J. Abrams, R. P. Ashley, L. C. Rowan, A. F. H. Goetz, and A. B. Kahle, "Mapping of hydrothermal alteration in the cuprite mining district, Nevada, using aircraft scanner images for the spectral region 0.46 to $2.36 \mu \mathrm{m}, "$ Geology, vol. 5, no. 12, pp. 713-718, 1977.

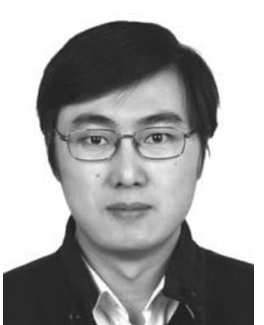

Lianru Gao (M'12) received the B.S. degree in civil engineering from Tsinghua University, Beijing, China, in 2002, and the Ph.D. degree in cartography and geographic information system from the Institute of Remote Sensing Applications, Chinese Academy of Sciences, Beijing, China, in 2007.

$\mathrm{He}$ is currently an Associate Professor with the Key Laboratory of Digital Earth Science, Institute of Remote Sensing and Digital Earth, Chinese Academy of Sciences. He has published over 60 papers in China and abroad. His research interests include spectral feature analysis, hyperspectral image processing, target detection, and image simulation.

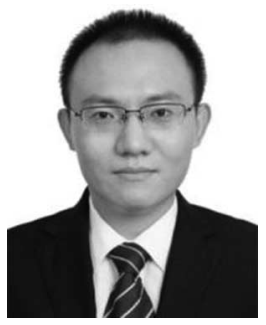

Jianwei Gao received the B.S. degree in computer science and technology from Beijing Jiaotong University, Beijing, China, in 2009, and the Ph.D. degree in cartography and geographic information system from the Institute of Remote Sensing and Digital Earth, Chinese Academy of Sciences, Beijing, China, in 2014.

$\mathrm{He}$ is currently an Associate Researcher with the Institute of Remote Sensing and Digital Earth, Chinese Academy of Sciences. His research interests include hyperspectral image processing, airborne remote sensing data processing, and high-performance parallel computing.

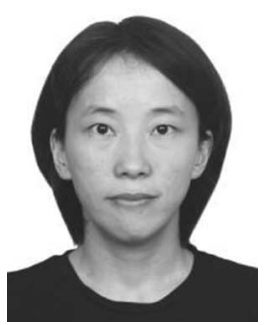

Jun Li (M'13) received the B.S. degree in geographic information systems from Hunan Normal University, Changsha, China, in 2004, the M.E. degree in remote sensing from Peking University, Beijing, China, in 2007, and the Ph.D. degree in electrical engineering from Universidade Técnica de Lisboa, Lisbon, Portugal, in 2011

From 2007 to 2011, she was a Marie Curie Research Fellow with the Departamento de Engenharia Electrotécnica e de Computadores and the Instituto de Telecomunicaes, Universidade Técnica de Lisboa, in the framework of the European Doctorate for Signal Processing (SIGNAL). She has also been actively involved in the Hyperspectral Imaging Network, a Marie Curie Research Training Network involving 15 partners in 12 countries and intended to foster research, training, and cooperation on hyperspectral imaging at the European level. Since 2011, she has been a Postdoctoral Researcher with the Hyperspectral Computing Laboratory, Department of Technology of Computers and Communications, Escuela Politécnica, University of Extremadura, Cáceres, Spain. Her research interests include hyperspectral image classification and segmentation, spectral unmixing, signal processing, and remote sensing.

Dr. Li has been a Reviewer of several journals, including the IEEE Transactions on Geoscience and Remote Sensing, the IEEE Geoscience And Remote Sensing Letters, Pattern Recognition, Optical Engineering, Journal of Applied Remote Sensing, and Inverse Problems and Imaging. She was the recipient of the 2012 Best Reviewer Award of the IEEE Journal of SElEcted TOPICS IN APPLIEd EARTh ObSERVATIONS AND REMOTE SENSING. 


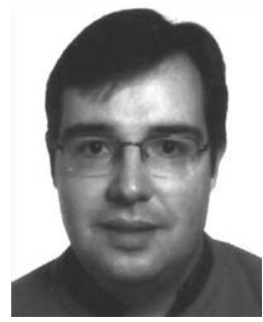

Antonio Plaza (M'05-SM'07-F'14) received the M.S. and Ph.D. degrees in computer engineering from the University of Extremadura, Caceres, Spain

He was a Visiting Researcher with the Remote Sensing Signal and Image Processing Laboratory, University of Maryland Baltimore County, Baltimore, MD, USA; the Applied Information Sciences Branch, Goddard Space Flight Center, Greenbelt, MD, USA; and the AVIRIS Data Facility, Jet Propulsion Laboratory, Pasadena, CA, USA. He is currently an Associate Professor with the Department of Technology of Computers and Communications, University of Extremadura, Caceres, Spain, where he is the Head of the Hyperspectral Computing Laboratory. He was the Coordinator of the Hyperspectral Imaging Network, which is a European project designed to build an interdisciplinary research community focused on hyperspectral imaging activities. He has been a Proposal Reviewer with the European Commission, the European Space Agency, and the Spanish Government. He has coedited a book on high-performance computing in remote sensing. He is the author or coauthor of around 300 publications on remotely sensed hyperspectral imaging, including more than 50 journal citation report papers, 20 book chapters, and over 200 conference proceeding papers. His research interests include remotely sensed hyperspectral imaging, pattern recognition, signal and image processing, and efficient implementation of large-scale scientific problems on parallel and distributed computer architectures.

Dr. Plaza was a Guest Editor for seven special issues on remotely sensed hyperspectral imaging for different journals, including the IEEE Transactions on Geoscience and Remote Sensing (for which he has served as an Associate Editor on hyperspectral image analysis and signal processing since 2007), the IEEE JOURNAL OF SELECTED TOPICS IN APPLIEd EARTh OBSERVATIONS AND REMOTE SENSING, the International Journal of High Performance Computing Applications, and the Journal of Real-Time Image Processing. He has served as a Reviewer for more than 280 manuscripts submitted to more than 50 different journals, including more than 140 manuscripts reviewed for the IEEE TRANSACTIONS ON GEOSCIENCE And Remote Sensing. He has served as a Chair for the IEEE Workshop on Hyperspectral Image and Signal Processing: Evolution in Remote Sensing in 2011. He has also been serving as a Chair for the Society of Photo-Optical Instrumentation Engineers (SPIE) Conference on Satellite Data Compression, Communications, and Processing since 2009 and for the SPIE Remote Sensing Europe Conference on High Performance Computing in Remote Sensing since 2011. He was the recipient of the recognition of Best Reviewers of the IEEE GEOSCIENCE AND REMOTE SENSING LETTERS in 2009 and the recognition of Best Reviewers of the IEEE TRANSACTIONS ON GEOSCIENCE AND REMOTE SENSING in 2010. He is currently serving as the Director of Education activities for the IEEE GEoscience ANd Remote SEnsing Society.

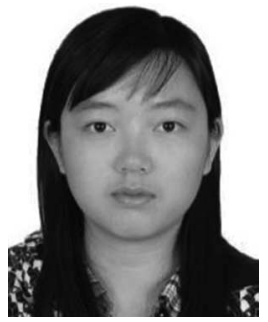

Lina Zhuang received two Bachelor's degrees in geographic information system and economics from South China Normal University, Guangzhou, China, in 2012. Currently, she is pursuing the M.S. degree at the Institute of Remote Sensing and Digital Earth, Chinese Academy of Sciences, Beijing, China.

Her research interests include hyperspectral image processing, pattern recognition, and machine learning.

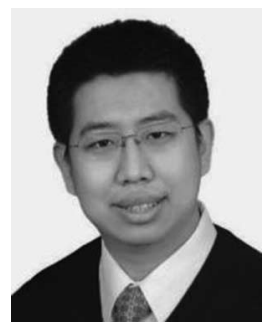

Xu Sun (M'12) received the B.S. degree in mathematics and application mathematics from Tsinghua University, Beijing, China, and the Ph.D. degree in cartography and geographical information system from the Graduate University of Chinese Academy of Sciences, Beijing China, in 2011.

$\mathrm{He}$ is currently an Associate Researcher with the Institute of Remote Sensing and Digital Earth, Chinese Academy of Sciences. His research interests include hyperspectral image processing and artificial intelligence algorithm.

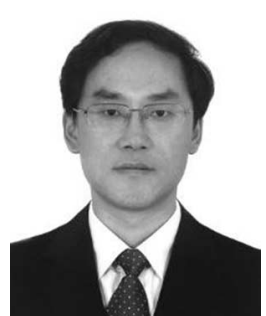

Bing Zhang (M'11-SM'12) received the B.S. degree in geography from Peking University, Beijing, China, the M.S. and Ph.D. degrees in remote sensing from the Institute of Remote Sensing Applications, Chinese Academy of Sciences (CAS), Beijing, China.

Currently, he is a Professor and the Deputy Director of the Institute of Remote Sensing and Digital Earth, CAS. He specializes in hyperspectral remote sensing and has more than 17 years of experience in studying and graduate education in this field. His research interests include development of physics-based models and image processing software for the use of hyperspectral remote sensing data in solving problems in geology, hydrology, ecology, and botany.

Prof. Zhang was the recipient of several Chinese National, Ministerial, and Provincial S\&T Progress Awards. 